\title{
Randomized phase II study of pemetrexed or pemetrexed plus bevacizumab for elderly patients with previously untreated non-squamous non-small cell lung cancer: Results of the Lung Oncology Group in Kyushu (LOGIK1201)
}

Minoru Fukuda ${ }^{1}$, Takeshi Kitazaki ${ }^{2}$, Daiki Ogawara ${ }^{3}$, Masao Ichiki ${ }^{4}$, Hiroshi Mukae ${ }^{5}$, Riichiroh Maruyama $^{6}$, Noriaki Nakagaki ${ }^{7}$, Midori Shimada ${ }^{2,5}$, Takaya Ikeda ${ }^{3,5}$, Junji Kishimoto $^{8}$, Taishi Harada ${ }^{9}$, Takashi Seto ${ }^{10}$, Noriyuki Ebi ${ }^{11}$, Koichi Takayama ${ }^{12}$, Isamu Okamoto $^{13}$, Yukito Ichinose ${ }^{14}$, Kenji Sugio ${ }^{15,16}$

${ }^{1}$ Clinical Oncology Center, Nagasaki University Hospital, Nagasaki, Japan; ${ }^{2}$ Division of Respiratory Diseases, Department of Internal Medicine, Japanese Red Cross Nagasaki Genbaku Hospital, Nagasaki, Japan; ${ }^{3}$ Department of Respiratory Medicine, Sasebo City General Hospital, Nagasaki, Japan; ${ }^{4}$ Department of Respirology, National Hospital Organization Kyushu Medical Center, Fukuoka, Japan; ${ }^{5}$ Department of Respiratory Medicine, Nagasaki University Graduate School of Biomedical Sciences, Nagasaki, Japan; ${ }^{6}$ Department of Thoracic Surgery, Omuta Tenryo Hospital, Omuta, Japan;

${ }^{7}$ Department of Respiratory Medicine, Steel Memorial Yawata Hospital, Kitakyushu, Japan; ${ }^{8}$ Center for Clinical and Translational Research, Kyushu University, Fukuoka, Japan; ${ }^{9}$ Department of Respiratory Medicine, Japan Community Health Care Organization (JCHO) Kyushu Hospital, Kitakyushu, Japan; ${ }^{10}$ Department of Thoracic Oncology, National Kyushu Cancer Center, Fukuoka, Japan; ${ }^{11}$ Department of Respiratory Oncology Medicine, Iizuka Hospital, Fukuoka, Japan; ${ }^{12}$ Department of Pulmonary Medicine, Kyoto Prefectural University of Medicine, Kyoto, Japan;

${ }^{13}$ Research Institute for Disease of the Chest, Graduate School of Medical Sciences, 
Kyushu University, Fukuoka, Japan; ${ }^{14}$ Clinical Research Institute, National Kyushu Cancer Center, Fukuoka, Japan; ${ }^{15}$ Department of Thoracic and Breast Surgery, Oita University Faculty of Medicine, Oita, Japan; ${ }^{16}$ Lung Oncology Group in Kyushu (LOGiK), Fukuoka, Japan

Short title: Phase II study of PEM vs. PEM/BEV

Address correspondence to: Minoru Fukuda, MD, $\mathrm{PhD}$

Clinical Oncology Center, Nagasaki University Hospital,

1-7-1 Sakamoto, Nagasaki, 852-8501, Japan

Phone: +81-(95)-819-7779; Fax:+81-(95)-819-7776; E-mail: mifukuda258@nifty.com

Keywords: pemetrexed, bevacizumab, lung cancer, elderly, NSqNSCLC 


\begin{abstract}
Objectives: To evaluate the efficacy and safety, we conducted a randomized phase II study of pemetrexed (Pem) versus Pem + bevacizumab (Bev) for elderly patients with non-squamous non-small cell lung cancer (NSqNSCLC).
\end{abstract}

Patients and methods: The eligibility criteria were as follows: NSqNSCLC, no prior therapy, stage IIIB/IV disease or postoperative recurrence, age: $\geq 75$ years, performance status (PS): 0-1, and adequate bone marrow function. The patients were randomly assigned (1:1 ratio) to receive Pem or Pem+Bev. The primary endpoint was progression-free survival (PFS). The secondary endpoints were the response rate, OS, toxicities, and cost-effectiveness.

Results: Forty-one patients were enrolled and 40 (20 from each group) were assessable. Their characteristics were as follows: male/female $=23 / 17$; median age $($ range $)=78(75-83) ;$ stage IIIB/IV/postoperative recurrence=1/30/9; PS 0/1=11/29. All cases involved adenocarcinoma. There was no significant intergroup difference in PFS and the median PFS (95\% confidence interval) values of the Pem and Pem+Bev groups were $5.4(3.0-7.4)$ and $5.5(3.6-9.9)$ months, respectively $(\mathrm{p}=0.66)$. The response rate was significantly higher in the Pem+Bev group ( $15 \%$ vs. $55 \%, p=0.0146)$, and there was no significant difference in OS (median: 16.0 vs. 16.4 months, $\mathrm{p}=0.58$ ). Grade 3 and 4 leukopenia, neutropenia, and thrombocytopenia were seen in 10 and 30, 20 and 55, and 5 and 5 cases, respectively. Drug costs were higher in the Pem+Bev group (median: $1,522,008$ vs. $3,368,428$ JPY, $\mathrm{p}=0.01)$. No treatment-related deaths occurred.

Conclusions: Adding Bev to Pem did not result in improved survival in the elderly NSqNSCLC patients. Compared with Pem+Bev, Pem monotherapy had similar effects on survival, a more favorable toxicity profile, and was more cost-effective in 
elderly NSqNSCLC patients. Pem monotherapy might be one of the optional regimen for NSqNSCLC patients aged $\geq 75$ years. 


\section{INTRODUCTION}

Lung cancer is the leading cause of cancer death worldwide. It is also the leading cause of cancer death in Japan, with 78,000 estimated deaths from lung cancer occurring in 2017 (20.6\% of all cancer deaths) (1), and non-small cell lung cancer (NSCLC) accounts for $80 \%$ of cases of lung cancer. Due to the aging of the Japanese population, the number of elderly patients with NSCLC in Japan is increasing. The frequency of severe toxicities is high in elderly patients treated with chemotherapy, even those that appear to have normal organ function (13). In elderly patients with advanced NSCLC, vinorelbine was reported to result in improved survival compared with supportive care alone (14), and docetaxel achieved even better survival outcomes than vinorelbine (15). Chemotherapy is approved as a treatment for elderly patients with NSCLC, and docetaxel monotherapy and vinorelbine monotherapy are recognized as standard regimens. However, effective and less toxic chemotherapy regimens are needed to improve the outcomes of elderly patients.

Pemetrexed is an antifolate, antitumor agent, which exerts its effects by interrupting folate-dependent metabolic processes that are essential for cell replication. It targets thymidylate synthase, dihydrofolate reductase, and glycinamide ribonucleotide formyltransferase (2). Compared with docetaxel monotherapy, pemetrexed exhibited equivalent efficacy against NSCLC and had significantly fewer side effects, and it was confirmed to be especially effective against non-squamous NSCLC (NSqNSCLC) (3). Subset analysis revealed that elderly patients aged $\geq 70$ years who received pemetrexed demonstrated longer time to progression (4.6 vs. 2.9 months) and overall survival (9.5 vs. 7.7 months) values than those treated with docetaxel (16). Thus, it seems that the milder toxicities of pemetrexed may lead to more favorable outcomes among elderly 
NSCLC patients.

Bevacizumab, a recombinant humanized IgG1 monoclonal antibody that binds to vascular endothelial growth factor, has become the focus of therapeutic interventions aimed at blocking tumor angiogenesis and elevating the blood levels of chemotherapeutic drugs by promoting tumor vascular stabilization (10-12). Bevacizumab has been approved for use in combination with the standard platinum-based chemotherapy or as a maintenance therapy after chemotherapy during the treatment of NSCLC patients without driver mutations (7-9), and it was found to significantly prolong overall survival and progression-free survival when added to first-line platinum-based chemotherapy in patients with advanced NSCLC (17). When added to the standard chemotherapy, there is a possibility that bevacizumab bring beneficial effects after excluding high-risk patients with squamous cell carcinoma or hemoptysis.

Therefore, compared with the standard chemotherapy pemetrexed/bevacizumab combination chemotherapy is considered to be a potentially useful treatment for elderly patients with NSqNSCLC. So, we conducted a randomized phase II study of pemetrexed versus pemetrexed plus bevacizumab treatment for elderly patients with NSqNSCLC. Both regimens were continued until tumor progression or unacceptable toxicities occurred.

\section{PATIENTS AND METHODS}

The study protocol was reviewed and approved by the protocol committee of the Lung Oncology Group in Kyushu (LOGiK), and the ethics committee of each institution. Written informed consent was obtained from all study participants. This 
study was an independent collaborative (unsponsored) group study. It was registered at the University Hospital Medical Information Network (UMIN) in Japan (registration number: UMIN000008771).

\section{Study design and patients}

LOGIK1201 was initially designed as a phase II randomized trial of pemetrexed versus pemetrexed plus bevacizumab as first-line treatments for patients with NSqNSCLC.

The eligibility criteria for this study were as follows: having a histologically and/or cytologically confirmed diagnosis of NSqNSCLC; having not received any previous chemotherapy except for molecularly targeted therapy or postoperative chemotherapy that was administered more than 6 months ago; not being indicated for radical radiotherapy; having clinical stage IIIB or IV disease or postoperative recurrence; not having any other active malignancies; being aged $\geq 75$ years old; having an Eastern Cooperative Oncology Group (ECOG) performance status (PS) of $\leq 1$; having adequate bone marrow function (a leukocyte count of $\geq 4,000 / \mu 1$, a hemoglobin level of $\geq 9.0 \mathrm{~g} / \mathrm{dl}$, and a platelet count of $\geq 10.0 \times 10^{4} / \mu \mathrm{l}$ ); having alanine aminotransferase (ALT) and aspartate transaminase (AST) levels of $<100$ IU/1, a serum bilirubin level of $\leq 1.5 \mathrm{mg} / \mathrm{dl}$, a serum creatinine level of $\leq 1.5 \mathrm{mg} / \mathrm{dl}$, a urinary protein score of $\leq 1+$, and an arterial $\mathrm{O}_{2}$ pressure of $\geq 60$ Torr or an $\mathrm{SpO}_{2}$ of $\geq 90 \%$; having a lesion that could be assessed using the Response Evaluation Criteria in Solid Tumors (RECIST), version 1.1; providing written informed consent; and having a life expectancy of $>3$ months. The exclusion criteria were as follows: having a symptomatic metastatic brain tumor, hemoptysis, arterial or venous thromboemboli, uncontrolled 
hypertension, perforation of the digestive tract, a severe tracheoesophageal fistula, peritoneal inflammation, congenital hemorrhagic diathesis, a coagulation system disorder, uncontrolled diabetes, emergency superior vena cava syndrome, symptomatic pericardial effusion, interstitial pneumonia (as determined by a chest X-ray), or medical problems that were severe enough to prevent compliance with the protocol; having undergone an operation within the last 28 days; or being scheduled for planned radiotherapy during the bevacizumab therapy. The threshold age of 75 for elderly patients was decided in our group because that the patients from 70 to 74 years old could receive platinum doublet for first line chemotherapy.

\section{Assessments}

Before treatment, the patients' complete medical histories were taken, and all patients underwent physical examinations, chest X-ray examinations, chest and abdominal computed tomography (CT) scans, radionuclide bone or positron emission tomography (PET)-CT scans, brain magnetic resonance imaging, and electrocardiography, as well as assessments of their complications, blood cell counts, blood biochemistry, urinary protein levels, and epidermal growth factor receptor (EGFR) mutations. In the assessment of cost-effectiveness, all of the main and supportive treatment drugs and blood infusions were included. Patient responses were assessed according to the RECIST, version 1.1. All adverse events were recorded and graded according to the Common Terminology Criteria for Adverse Events (CTCAE), version 4.0. The interval between CT scans for follow-up were at least once every 4 to 6 weeks. 


\section{Treatment}

The patients were randomly allocated with equal probability (1:1) to receive pemetrexed or pemetrexed plus bevacizumab, and were stratified by disease stage (IIIB/IV/postoperative recurrence) and sex (male/female). The patients received chemotherapy with pemetrexed $\left(500 \mathrm{mg} / \mathrm{m}^{2}\right.$; a 10 -minute intravenous infusion; every 21 days) or pemetrexed plus bevacizumab (15 mg/kg; an intravenous infusion; every 21 days) until one of the criteria for treatment removal was met, which included disease progression based on the investigator's assessment or symptomatic deterioration; a treatment delay of longer than 2 weeks; pemetrexed dose reduction to $<400 \mathrm{mg} / \mathrm{m}^{2}$; bevacizumab dose reduction to $<10 \mathrm{mg} / \mathrm{kg}$; drug-induced interstitial pneumonia; bleeding episodes, such as hemoptysis, in the bevacizumab-treated patients; or unacceptable toxicity. Treatment was postponed if the patient's leukocyte count fell to $<3,500 / \mu 1$, their neutrophil count fell to $<1,500 / \mu 1$, or their platelet count fell to $<10 \mathrm{x}$ $10^{4} / \mu \mathrm{l}$; a fever of $\geq 38.0^{\circ} \mathrm{C}$ combined with infection occurred; the patient's AST/ALT level rose to $>2.5$ times above the upper normal limit; the patient's serum creatinine level increased to $>1.5$ times above the upper normal limit; heart/lung toxicities of grade $\geq 2$ occurred; or other toxicities of $\geq$ grade 3 , except for hair loss, nausea/vomiting, appetite loss, and fatigue, occurred. Dose reduction to a pemetrexed dose of $400 \mathrm{mg} / \mathrm{m}^{2}$ and/or a bevacizumab dose of $10 \mathrm{mg} / \mathrm{kg}$ was performed if any of the following adverse events were observed: a neutrophil count of $<500 / \mu 1$, a neutrophil count of $<1,000 / \mu 1$ with an infra-axillary fever of $37.5^{\circ} \mathrm{C}$ (oral cavity: $38.0^{\circ} \mathrm{C}$ ), a platelet count of $<5 \mathrm{x}$ $10^{4} / \mu 1$, or grade $\geq 3$ non-hematological toxicities in previous cycles.

\section{Statistical analysis}


The primary endpoint of this study was progression-free survival, which was assessed by an independent review committee. The secondary endpoints included overall survival, the tumor response, toxicities, and cost-effectiveness. The expected median progression-free survival times of the pemetrexed group (4) and the pemetrexed plus bevacizumab group (18) were 3.3 months and 7.4 months, respectively. The enrollment period was 5 years long, the follow-up period was 1 year long, the significance level was set at 0.2 , and the power level was set at 0.8 for the log-rank test. Therefore, the estimated required number of patients was more than 32. As some cases would be ineligible, the target sample size was set at 40 (20 for each group). In the survival analysis, survival curves were produced using the Kaplan-Meier method, and the stratified log-rank test, which stratified the data according to the allocation factors (disease stage and sex), was used for comparisons between groups. Furthermore, the stratified proportional hazards model, which stratified the data according to the allocation factors, was used to estimate hazard ratios. The confidence intervals for the median survival time and survival rate were computed using the Brookmeyer and Crowley method and Greenwood formula, respectively. A stratified multiple logistic regression model, which considered the stratified factors, was used for comparisons of binary indefinite numbers, including the response rate. The confidence intervals for the response rate were computed using the Wilson method. The Wilcoxon rank sum test was used for the analysis of cost-effectiveness. In the main analysis, $p$-values of $<0.2$ (two-sided) were defined as statistically significant. In the other analyses, p-values of $<0.05$ (two-sided) were regarded as statistically significant. No adjustments for multiplicity were made. SAS Ver.9.3 was used for all statistical analyses. 


\section{RESULTS}

Forty-one patients from 12 institutions were enrolled and underwent randomization (20 to the pemetrexed group and 21 to the pemetrexed plus bevacizumab group) in this trial between August 31, 2012, and June 30, 2016. The data cut-off date was October 25, 2017. A consort diagram is shown in Supplement Figure 1. Treatment was discontinued before the main treatment was administered in one patient in the pemetrexed plus bevacizumab group because of drug eruptions caused by the premedication. Survival, the response to treatment, and toxicity were evaluated in the remaining 40 patients as the full analysis set (FAS). The patients' baseline demographic and clinical characteristics were balanced across the two groups, as shown in Table 1. Patients between the ages of 75 and 83 were enrolled, and their median age was 78 years. All of the tumors were histologically diagnosed as adenocarcinoma.

\section{Treatment administration}

The median duration of treatment was 3.0 months (range, 0.7 to 14.9 months) in the pemetrexed group and 3.9 months (range, 0.9 to 23.6 months) in the pemetrexed plus bevacizumab group. A total of 123 cycles of pemetrexed were administered, with the median number of cycles administered per patient being 4.5 (range, 1 to 21 cycles), and a total of 157 cycles of pemetrexed plus bevacizumab were administered, with the median number of cycles administered per patient being 5.5 (range, 2 to 31 cycles). Dose reduction was performed in 3 patients $(15 \%)$ and 13 cycles $(11 \%)$ in the pemetrexed group and in 6 patients $(30 \%)$ and 16 cycles $(10 \%)$ in the pemetrexed plus bevacizumab group. The reasons for treatment discontinuation included tumor progression $(n=22,55 \%)$, adverse events $(n=7,18 \%)$, treatment postponement for more 
than 14 days $(n=3,8 \%)$, patient refusal $(n=2,5 \%)$, and other considerations of the attending physician $(n=6,15 \%)$. Number of treatment cycles was shown in Supplement Figure 2.

\section{Efficacy}

Progression-free survival

The progression-free survival times of the 40 patients are shown in Figure 1A. The median duration of the progression-free survival period [ $95 \%$ confidence interval (CI)] was $5.4(3.0-7.4)$ months in the pemetrexed group and 5.5 (3.6-9.9) months in the pemetrexed plus bevacizumab group. There was no significant difference in the progression-free survival time between the two groups ( $\mathrm{p}=0.66$, log-rank test). According to the stratified Cox proportional hazards model, the hazard ratio $(95 \% \mathrm{CI})$ for the pemetrexed plus bevacizumab group versus the pemetrexed group was 0.84 (0.38-1.86). The 6-month progression-free survival rate $(95 \% \mathrm{CI})$ was $45.5 \%$ $(21.0-67.1 \%)$ in the pemetrexed group and $50.0 \%(27.1-69.2 \%)$ in the pemetrexed plus bevacizumab group.

\section{Overall survival}

The overall survival data for the 40 patients are shown in Figure 1B. The median overall survival time $(95 \% \mathrm{CI})$ was $16.0(9.0-29.1)$ months in the pemetrexed group and 16.4 (7.9-36.0) months in the pemetrexed plus bevacizumab group. There was no significant difference in the overall survival time between the two groups ( $p=0.58$, log-rank test). The stratified Cox proportional hazards model indicated that the hazard ratio $(95 \% \mathrm{CI})$ for the pemetrexed plus bevacizumab group versus the 
pemetrexed group was $0.79(0.35-1.79)$. The one-year overall survival rate $(95 \% \mathrm{CI})$ was $62.9 \%(37.2-80.4 \%)$ in the pemetrexed group and $74.7 \%(49.4-88.6 \%)$ in the pemetrexed plus bevacizumab group. The two-year overall survival rate $(95 \% \mathrm{CI})$ was $32.7 \%(10.4-57.5 \%)$ in the pemetrexed group and $40.4 \%(18.6-61.5 \%)$ in the pemetrexed plus bevacizumab group.

\section{Response rate}

The median amount of tumor shrinkage $(95 \% \mathrm{CI})$ was $15.0 \%(5.2-36.0 \%)$ in the pemetrexed group and 55.0\% (34.2-74.2\%) in the pemetrexed plus bevacizumab group. The best overall responses in each group were as follows: complete response (CR), 0; partial response (PR), 3; stable disease (SD), 11; progressive disease (PD), 4; and not evaluable (NE), 2 in the pemetrexed group and CR, 0; PR, 11; SD, 7; PD, 1; and NE, 1 in the pemetrexed plus bevacizumab group (Table 2). The best tumor shrinkage rate and best overall responses seen in the 39 patients are shown in Supplement Figure 3. One patient whose response was NE in the pemetrexed group could not be monitored and so was excluded. Intergroup comparisons were conducted using a stratified logistic regression model, in which the data were stratified according to disease stage and sex. The response rate of the pemetrexed plus bevacizumab group was significantly higher than that of the pemetrexed group $(\mathrm{p}=0.0146)$.

\section{Safety and adverse events}

The hematological and non-hematological toxicities experienced by the patients in each group are listed in Table 3. Grade 3/4 neutropenia, diarrhea, and proteinuria tended to occur more frequently in the pemetrexed plus bevacizumab group 
than in the pemetrexed group, but the differences were not significant. Oral mucositis and hyponatremia were observed significantly higher in the pemetrexed plus bevacizumab group compare with pemetrexed group ( $25 \%$ vs. $0 \%, p=0.047$, and $90 \%$ vs. $50 \%, p=0.014$, respectively). The frequencies of the other toxicities did not differ between the two groups. One patient with nasal bleeding of grade 1 was observed in the pemetrexed plus bevacizumab group. There were no cases of grade $3 / 4$ pneumonitis or treatment-related deaths.

\section{Cost-effectiveness}

There was no difference in the progression-free survival or overall survival time between the two groups. Therefore, the total medication costs (for pemetrexed, bevacizumab, and concomitant medication), and the individual costs for pemetrexed, bevacizumab, and concomitant medication were calculated for each group, and then intergroup comparisons were performed. The total cost of treatment in the pemetrexed plus bevacizumab group (median: 3,368,428 Japanese Yen (JPY); range: 1,074,314 to $18,670,494$ JPY) was significantly higher than the total cost of treatment in the pemetrexed group (median: 1,522,008 JPY; range: 318,118 to $7,211,480 \mathrm{JPY})(\mathrm{p}=0.01)$. As there were no differences in the cost of pemetrexed or the concomitant medication between the two groups, the intergroup cost differences were mainly due to the cost of bevacizumab.

\section{Relationship between tumor shrinkage and progression-free survival}

The relationships between the tumor shrinkage rate (during the best tumor response) and the progression-free survival rate in the two groups are shown in Figure 2. 
One patient in the pemetrexed plus bevacizumab group had both a high shrinkage rate and a long progression-free survival period. The other patients in the pemetrexed plus bevacizumab group tended to have high shrinkage rates, but this did not result in longer survival.

\section{Subpopulation analyses of progression-free and overall survival}

In the subpopulation analyses of progression-free and overall survival according to sex, age, disease stage, EGFR mutation status, and smoking history, no significant differences were detected between the treatment groups (Figure 3).

\section{DISCUSSION}

The present study is the first prospective study to compare the clinical impact of pemetrexed plus bevacizumab with that of pemetrexed in NSqNSCLC patients aged $\geq 75$ years. Compared with pemetrexed treatment, pemetrexed plus bevacizumab did not result in a longer progression-free survival period (the primary endpoint). In addition to having similar effects on survival to pemetrexed plus bevacizumab, pemetrexed monotherapy exhibited a more favorable toxicity profile and was more cost-effective in NSqNSCLC patients aged $\geq 75$ years, and so it seems to be a useful treatment option for this population.

The current standard treatments for elderly NSCLC patients are vinorelbine or docetaxel, and docetaxel was found to be superior in a comparison between these two agents $(6,14)$. About combination chemotherapy for elderly NSCLC, carboplatin and weekly paclitaxel was found to be superior with monotherapy (19), however, was also reported severe toxicity with $4.4 \%$ toxic deaths. Cisplatin plus docetaxel failed to 
demonstrate any survival advantage compare with docetaxel monotherapy (20). Pemetrexed is a more effective treatment for NSqNSCLC than docetaxel and causes significantly fewer adverse events $(3,5)$, and adding bevacizumab to the standard chemotherapy is expected to have additional beneficial effects against NSqNSCLC. Therefore, a number of prospective studies of pemetrexed or pemetrexed plus bevacizumab for elderly NSqNSCLC patients have been conducted, as shown in Supplement Table $1(4,21-23)$. Pemetrexed plus bevacizumab tended to produce better outcomes than pemetrexed in terms of the response rate (25-55\% vs. $13-25 \%)$ and progression-free survival (4.8-5.5 months vs. 3.3-5.4 months). On the other hand, pemetrexed tended to produce better outcomes than pemetrexed plus bevacizumab in terms of overall survival (16.0-18.2 months vs. 11.6-16.4 months). One possible reason for these findings is that the combination chemotherapy was too toxic for elderly patients because of their decreased physical fitness and organ function and so did not result in longer overall survival. However, pemetrexed tends to be associated with a higher frequency of grade 3 or 4 neutropenia (29-39\% vs. $21-25 \%$ ) than pemetrexed plus bevacizumab, although that was not the case in the present study, and it is difficult to compare the outcomes of prospective studies conducted in different situations; therefore, a randomized controlled study is needed.

In the present study, which directly compared the efficacy of pemetrexed and pemetrexed plus bevacizumab, the frequencies of grade 3 or 4 neutropenia were higher in the pemetrexed plus bevacizumab group (20\% and 55\%, respectively), which indicated that the combination treatment had stronger side effects than the monotherapy. Such direct intergroup comparisons are important. Thus, our study is meaningful, as it is 
the first to directly compare the outcomes of pemetrexed and pemetrexed plus bevacizumab.

Regarding progression-free survival, we did not detect any difference between the two groups. So, why was there no intergroup difference in progression-free survival? In the current study, the case groups were designed based on the assumption that the expected median progression-free survival periods of the pemetrexed group and pemetrexed plus bevacizumab group were 3.3 months and 7.4 months, respectively; however, the actual median progression-free survival period was 5 to 6 months in both groups. As shown in Figure 3, the combination treatment caused tumor shrinkage, which seemed to lead to an extension of the progression-free survival period. Looking at the relationship between tumor shrinkage and progression-free survival, which is shown in Figure 4, marked tumor shrinkage and prolonged progression-free survival were seen in only one case in the pemetrexed plus bevacizumab group seems to be more of an outlier, and tumor shrinkage was not linked to longer progression-free survival in almost cases in this group. The current study was still a small number study with only 40 patients, therefore, the result of our study is not deterministic and it is weak point of the study.

With respect to combination treatment involving platinum doublet chemotherapy and bevacizumab in elderly NSqNSCLC patients, no additional effects were observed in patients aged $\geq 70$ years in the subset analysis conducted in the ECOG4599 trial, and more cases of grade 3 or 4 neutropenia, bleeding, and proteinuria were seen in these patients than in those aged $<70(24)$. In a subset analysis that combined the ECOG4599 and PointBreak trials, bevacizumab tended to have weak additional effects on OS and progression-free survival, especially in patients aged $>75$ 
years (25). Although these findings relate to the additional effects of adding bevacizumab to platinum chemotherapy, according to the subset analyses of the clinical trials conducted to date it seems that bevacizumab has weak additional effects in elderly NSqNSCLC patients aged $\geq 75$ years, which agrees with the results of our trial. In recent reports, Wakelee HA et al. conducted large randomized phase III trial with or without bevacizumab for adjuvant chemotherapy of resected NSCLC (E1505), and addition of bevacizumab did not improve overall survival both in all and elderly $(>60)$ cases (26). Whereas Xing $\mathrm{P}$ et al. suggest bevacizumab containing regimen have clinical benefits over a non-bevacizumab regimen in real world study of NSqNSCLC including elderly patients (27), and it is also required to carefully evaluate the adaptation of bevacizumab from now.

Regarding toxicities, leukopenia and neutropenia (bone marrow toxicities) tended to exhibit high frequencies in the pemetrexed plus bevacizumab group. Neutropenia was seen in $80 \%$ of cases, and it was classified as grade 3 or 4 in $55 \%$ of these cases; therefore, attention must be paid to reducing the risk of neutropenia when bevacizumab is administered to elderly patients. Bleeding tendency and proteinuria, which are characteristics of bevacizumab treatment, did not occur at high frequencies. Stomatitis was not observed in any case in the pemetrexed group, whereas it was seen in 5 patients $(25 \%)$ in the pemetrexed plus bevacizumab group. One of these cases was classified as grade 1 for the first and second cycles, but it worsened to grade 3 in the third cycle, and grade 3 anorexia and fatigue occurred concomitantly, which resulted in treatment termination in the third cycle. Thus, stomatitis might be an important adverse event although it was not examined in other studies in which pemetrexed plus bevacizumab was used to treat elderly patients with $\operatorname{NSqNSCLC}(22,23)$. 
Regarding cost-effectiveness which was secondary endpoint, the total cost of treatment in the pemetrexed plus bevacizumab group was significantly higher than the total cost of treatment in the pemetrexed group. Because the survival data of two groups were similar, it is important point and seems to help our determination in practical treatment.

In conclusion, this was the first prospective randomized study to compare the clinical impact of pemetrexed plus bevacizumab with that of pemetrexed in NSqNSCLC patients aged $\geq 75$ years. Adding bevacizumab to pemetrexed did not result in improved survival in the elderly NSqNSCLC patients. In fact, compared with pemetrexed plus bevacizumab, pemetrexed monotherapy had similar effects on survival, but displayed a more favorable toxicity profile and was more cost-effective, and thus, might be one of the optional regimen for NSqNSCLC patients aged $\geq 75$ years.

\section{Acknowledgements}

We thank the Clinical Research Support Center Kyushu for their support with the monitoring and data management and Takayuki Shimose for his help with the data analyses. The result of LOGIK1201 was presented in IASLC $19^{\text {th }}$ World Conference on Lung Cancer, September 23-26, 2018, Toronto, Canada.

\section{CONFLICTS OF INTEREST STATEMENT}

MF, HM, TS, KT, and IO received an honoraria from Eli Lilly and Chugai Pharma. MF and HM received research funding from Eli Lilly, and TS, KT, and IO received research funding from Eli Lilly and Chugai Pharma. The other authors have no conflicts of interest to declare. 


\section{REFERENCES}

1. National Cancer Center Japan, Center for Cancer Control and Information Services. [Cited 14 November 2017]. Available from http://ganjoho.jp/reg_stat/statistics/stat/short_pred.html

2. R. M. Schultz, V. F. Patel,, J. F. Worzalla, et al. Role of thymidylate synthase in the antitumor activity of the multitargeted antifolate, LY231514. Anticancer Res. 19 (1999) 437-443.

3. N. Hanna, F. A. Shepherd, F. V. Fossella, et al. Randomized phase III trial of pemetrexed versus docetaxel in patients with non-small-cell lung cancer previously treated with chemotherapy. J. Clin. Oncol. 22 (2004) 1589-1597.

4. Y. H. Kim, M. Hirabayashi, S. Kosaka, et al. Phase II study of pemetrexed as first-line treatment in elderly $(\geq 75)$ non-squamous non-small-cell lung cancer: Kyoto Thoracic Oncology Research Group Trial 0901. Cancer Chemother. Pharmacol. 71 (2013) 1445-1451.

5. G. Scagliotti, N. Hanna, F. Fossela, et al. The differential efficacy of pemetrexed according to NSCLC histology: A review of two phase III studies. Oncologist 14 (2009) 253-263.

6. S. Kudoh, K. Takeda, K. Nakagawa, et al. Phase III study of docetaxel compared with vinorelbine in elderly patients with advanced non-small-cell lung cancer: Results of the West Japan Thoracic Oncology Group Trial (WJTOG 9904). J. Clin. Oncol. 24 (2006) 3657-3663.

7. A. Sandler, R. Gray, M. C. Perry, et al. Paclitaxel-carboplatin alone or with bevacizumab for non-small-cell lung cancer. N. Engl. J. Med. 355 (2006) 2542-2550. 
8. M. Reck, J. von Pawel, P. Zatloukal, et al. Phase III trial of cisplatin plus gemcitabine with either placebo or bevacizumab as first-line therapy for nonsquamous non-small-cell lung cancer: AVAil. J. Clin. Oncol. 27 (2009) 1227-1234.

9. R. Petrioli, E. Francini, A. I. Fiaschi, et al. Switch maintenance treatment with oral vinorelbine and bevacizumab after induction chemotherapy with cisplatin, gemcitabine and bevacizumab in patients with advanced non-squamous non-small cell lung cancer: a phase II study. Med. Oncol. 32 (2015) 134.

10. J. Jenab-Wolcott, \& B. J. Giantonio. Bevacizumab: current indications and future development for management of solid tumors. Expert Opin. Biol. Ther. 9 (2009) $507-517$.

11. A. T. Byrne, L. Ross, J. Holash, et al. Vascular endothelial growth factor-trap decreases tumor burden, inhibits ascites, and causes dramatic vascular remodeling in an ovarian cancer model. Clin. Cancer Res. 9 (2003) 5721-5728.

12. C. G. Willett, Y. Boucher, E. di Tomaso, et al. Direct evidence that the VEGF-specific antibody bevacizumab has antivascular effects in human rectal cancer. Nat. Med. 10 (2004) 145-147.

13. F. Oshita, T. Kurata, T. Kasai, et al. Prospective evaluation of the feasibility of cisplatin based chemotherapy for elderly lung cancer patients with normal organ functions. Jpn. J. Cancer Res. 86 (1995) 1198-1202.

14. The Elderly Lung Cancer Vinorelbine Italian Study Group. Effect of vinorelbine on quality of life and survival of elderly patients with advanced non-small cell lung cancer. J. Natl. Cancer Inst. 91 (1999) 66-72.

15. S. Kudoh, K. Takeda, K. Nakagawa, et al. Phase III study of docetaxel compared with vinorelbine in elderly patients with advanced non-small-cell lung cancer: 
results of the West Japan Thoracic Oncology Group Trial (WJTOG 9904). J. Clin. Oncol. 24 (2006) 3657-3663.

16. G. J. Weiss, C. Langer, R. Rosell, et al. Elderly patients benefit from second-line cytotoxic chemotherapy: a subset analysis of a randomized phase III trial of pemetrexed compared with docetaxel in patients with previously treated advanced non-small-cell lung cancer. J. Clin. Oncol. 24 (2006) 4405-4411.

17. J. C. Soria, A. Mauguen, M. Reck, et al. Systematic review and meta-analysis of randomised, phase II/III trials adding bevacizumab to platinum-based chemotherapy as first-line treatment in patients with advanced non-small-cell lung cancer. Ann. Oncol. 24 (2013) 20-30.

18. F. Bariesi, A. Scherpereel, V. Gorbunova, et al. Maintenance bevacizumab-pemetrexed after first-line cisplatin-pemetrexed-bevacizumab for advanced nonsquamous nonsmall-cell lung cancer: updated survival analysis of the AVAPERL (MO22089) randomized phase III trial. Ann. Oncol. 25 (2014) 1044-1052.

19. E. Quoix, G. Zalcman, J. P. Oster, et al. Carboplatin and weekly paclitaxel doublet chemotherapy compared with monotherapy in elderly patients with advanced non-small-cell lung cancer: IFCT-0501 randomised, phase 3 trial. Lancet 378 (2011) 1079-1088.

20. T. Abe, K. Takeda, Y. Ohe, et al. Randomized phase III trial comparing weekly docetaxel plus cisplatin versus docetaxel monotherapy every 3 weeks in elderly patients with advanced non-small-cell lung cancer: the intergroup trial JCOG0803/WJOG4307L. J. Clin. Oncol. 33 (2015) 575-581.

21. Y. Hattori, M. Iwasaku, M. Satouchi, et al. A phase II study of pemetrexed in 
chemotherapy-naïve elderly patients aged $\geq 75$ years with advanced non-squamous non-small-cell lung cancer (HANCHIN Oncology Group 003). Jpn. J. Clin. Oncol. 43 (2013) 1184-1189.

22. T. Kozuki, N. Nogami, H. Kitajima, et al. Feasibility study of first-line chemotherapy using pemetrexed and bevacizumab for advanced or recurrent nonsquamous non-small cell lung cancer in elderly patients: TORG1015. BMC Cancer 16 (2016) 306.

23. W. Schuette, C. P. Schneider, W. Engel-Riedel, et al. 65plus: open-label study of bevacizumab in combination with pemetrexed or pemetrexed/carboplatin as first-line treatment of patients with advanced or recurrent nonsquamous non-small-cell lung cancer. Lung Cancer 8 (2017) 217-229.

24. S. S. Ramalingam, S. E. Dahlberg, C. J. Langer, et al. Outcomes for elderly, advanced-stage non small-cell lung cancer patients treated with bevacizumab in combination with carboplatin and paclitaxel: analysis of Eastern Cooperative Oncology Group Trial 4599. J. Clin. Oncol. 26 (2008) 60-65.

25. C. J. Langer, M. A. Socinski, J. D. Patel, et al. Isolating the role of bevacizumab in elderly patients with previously untreated nonsquamous non-small cell lung cancer: secondary analyses of the ECOG 4599 and Pointbreak trials. Am. J. Clin. Oncol. 39 (2016) 441-447.

26. Wakelee HA, Dahlberg SE, Keller SM, et al. Adjuvant chemotherapy with or without bevacizumab in patients with resected non-small-cell lung cancer (E1505): an open-lavel, multicenter, randomized, phase 3 trial. Lancet Oncol. 18 (2017) $1610-1623$.

27. Xing $\mathrm{P}, \mathrm{Mu} \mathrm{Y}$, Wang $\mathrm{Y}$, et al. Real world study of regimen containing bevacizumab 
as first-line therapy in Chinese patients with advanced non-small cell lung cancer.

Thorac. Cancer 9 (2018) 805-813. 
Figure 1A

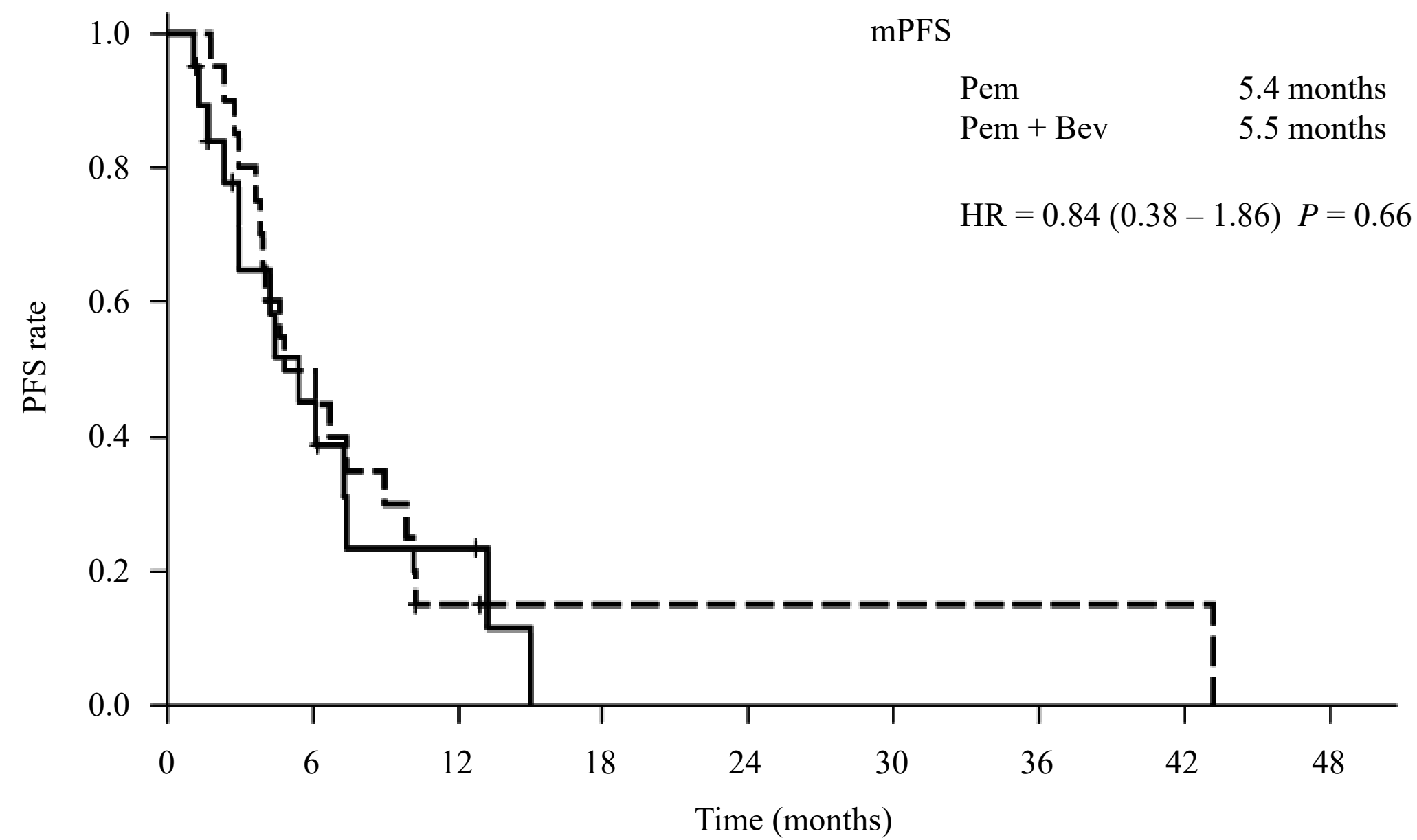

\section{No. of patients at risk}

Pem

20

7
10

3
2

0

0
1

$\begin{array}{ll}0 & 0 \\ 1 & 1\end{array}$

$\begin{array}{ll}0 & 0 \\ 1 & 0\end{array}$




\section{Figure 1B}

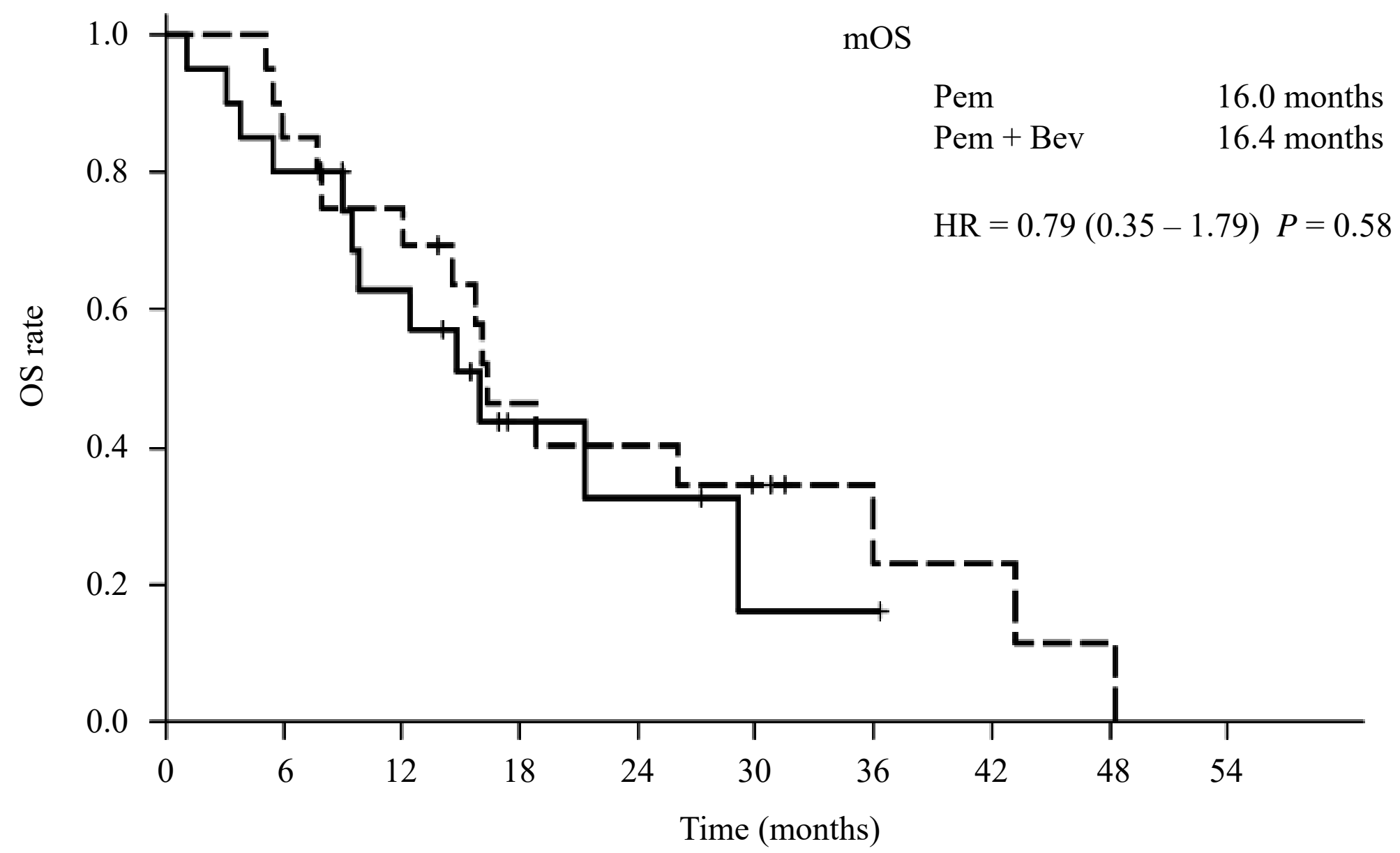

No. of patients at risk

$\begin{array}{lllllllllll}\text { Pem } & 20 & 16 & 11 & 4 & 3 & 1 & 1 & 0 & 0 & 0 \\ \text { Pem }+ \text { Bev } & 20 & 17 & 14 & 8 & 7 & 5 & 3 & 2 & 1 & 0\end{array}$


Figure 2
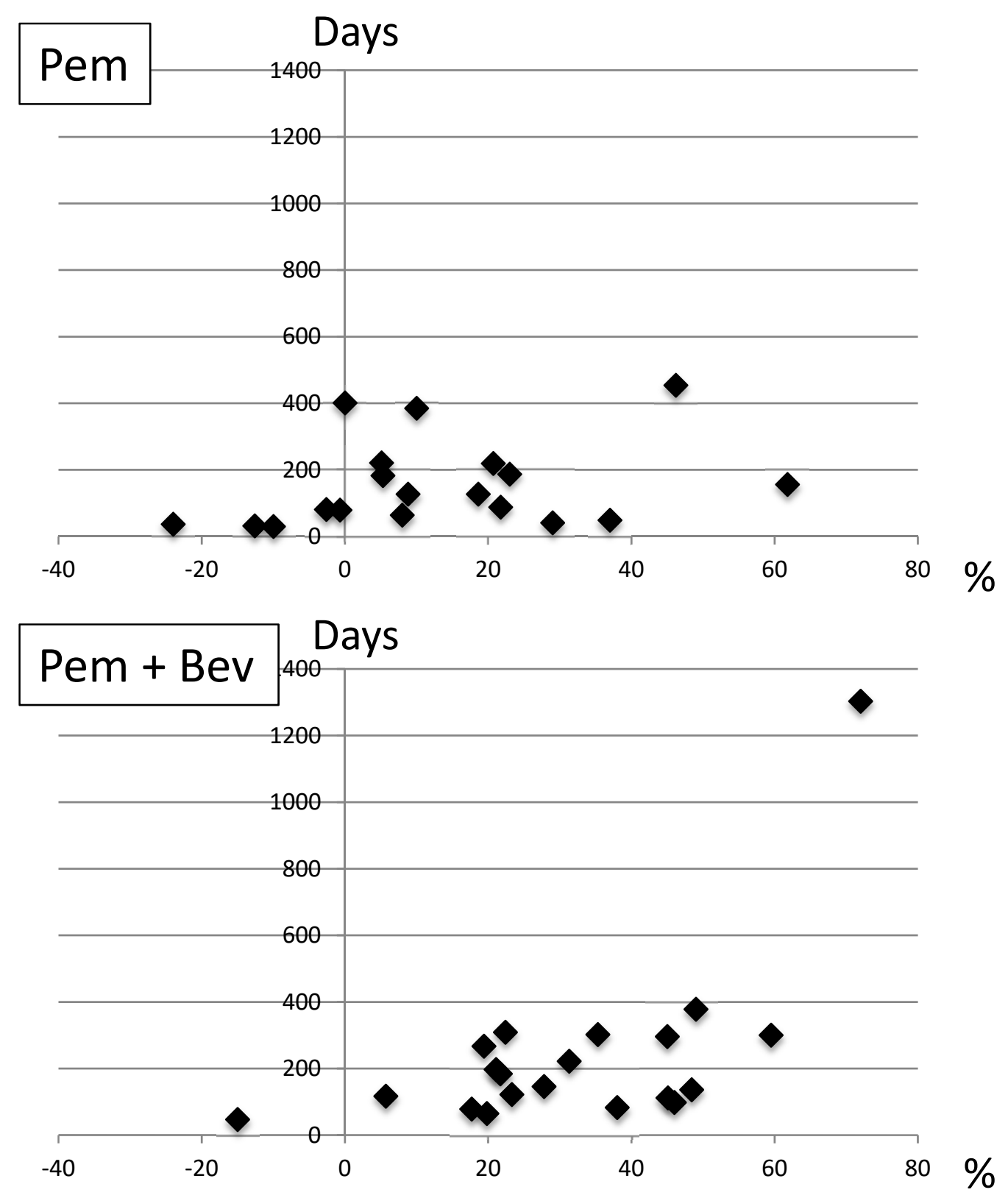


\section{Figure 3A}

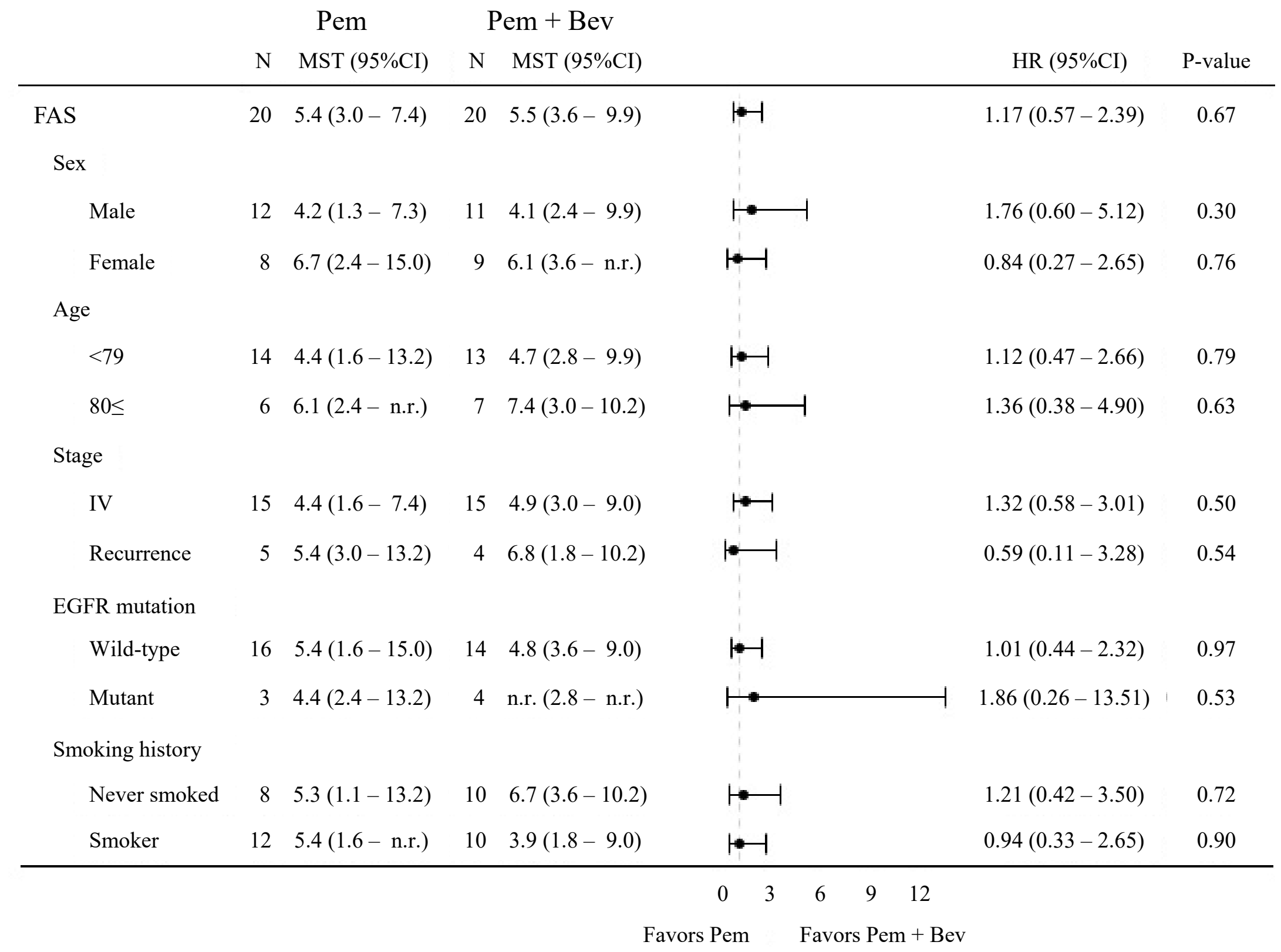




\section{Figure 3B}

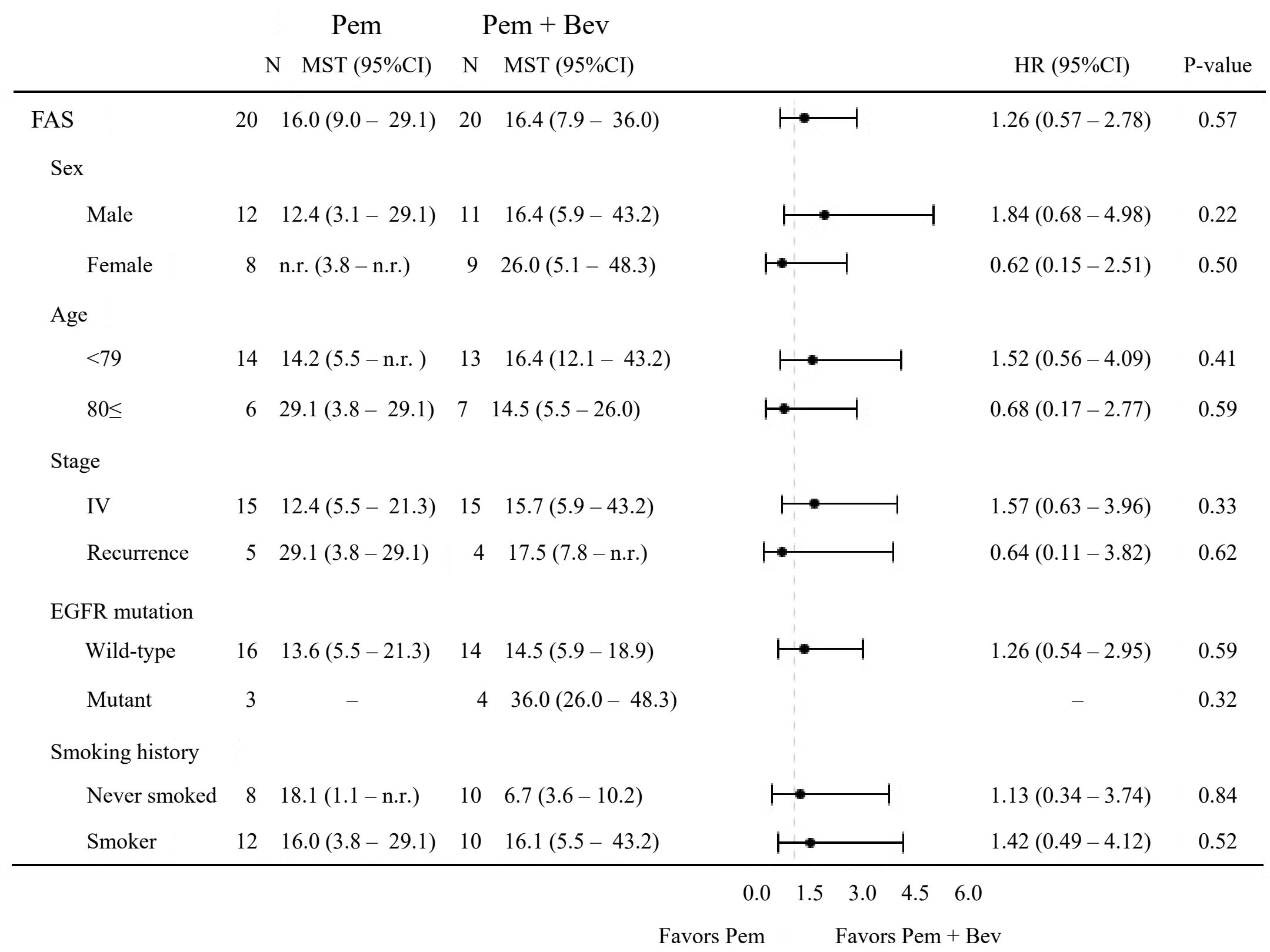


Table 1. Patient characteristics

\begin{tabular}{|c|c|c|c|c|c|c|}
\hline \multirow[t]{3}{*}{ Characteristics } & \multicolumn{2}{|c|}{ Pem } & \multicolumn{2}{|c|}{ Pem + Bev } & \multicolumn{2}{|c|}{ Total } \\
\hline & \multicolumn{2}{|c|}{$\mathrm{N}=20$} & \multicolumn{2}{|c|}{$\mathrm{N}=20$} & \multicolumn{2}{|c|}{$\mathrm{N}=40$} \\
\hline & \multicolumn{2}{|c|}{ number $(\%)$} & \multicolumn{2}{|c|}{ number $(\%)$} & \multicolumn{2}{|c|}{ number $(\%)$} \\
\hline \multicolumn{7}{|l|}{ Gender } \\
\hline male & 12 & $(60)$ & & $(55)$ & 23 & $(58)$ \\
\hline female & & $(40)$ & 9 & (45) & 17 & (43) \\
\hline \multicolumn{7}{|l|}{ Age (years) } \\
\hline median & \multicolumn{2}{|c|}{77.5} & \multicolumn{2}{|c|}{78.5} & \multicolumn{2}{|c|}{78.0} \\
\hline range & \multicolumn{2}{|c|}{$75-82$} & \multicolumn{2}{|c|}{$75-83$} & \multicolumn{2}{|c|}{$75-83$} \\
\hline \multicolumn{7}{|l|}{ Stage } \\
\hline IIIB & 0 & $(0)$ & 1 & $(5)$ & 1 & $(3)$ \\
\hline IV & 15 & $(75)$ & 15 & (75) & 30 & (75) \\
\hline postope recurrence & 5 & $(25)$ & 4 & (20) & 9 & (23) \\
\hline \multicolumn{7}{|l|}{ PS } \\
\hline 0 & 5 & $(25)$ & 6 & $(30)$ & 11 & $(28)$ \\
\hline 1 & 15 & $(75)$ & 14 & $(70)$ & 29 & (73) \\
\hline \multicolumn{7}{|l|}{ Histology } \\
\hline adenocarcinoma & 20 & $(100)$ & 20 & $(100)$ & 40 & $(100)$ \\
\hline large cell carcinoma & 0 & $(0)$ & 0 & $(0)$ & 0 & (0) \\
\hline others & 0 & $(0)$ & 0 & $(0)$ & 0 & $(0)$ \\
\hline \multicolumn{7}{|l|}{ EGFR mutation* } \\
\hline yes & 3 & $(15)$ & 4 & $(20)$ & 30 & $(18)$ \\
\hline no & 16 & $(80)$ & 14 & (70) & 7 & $(75)$ \\
\hline unknown & 1 & $(5)$ & 2 & (10) & 3 & $(8)$ \\
\hline \multicolumn{7}{|l|}{ Smoking history } \\
\hline yes & 12 & $(60)$ & 10 & $(50)$ & 22 & $(55)$ \\
\hline no & 8 & $(40)$ & 10 & $(50)$ & 18 & $(45)$ \\
\hline \multicolumn{7}{|l|}{$\operatorname{GFR}\left(\mathrm{ml} / \mathrm{min} .1 .73 \mathrm{~m}^{2}\right)$} \\
\hline median & \multirow{2}{*}{\multicolumn{2}{|c|}{$\begin{array}{c}63 \\
37-120\end{array}$}} & \multicolumn{2}{|c|}{72} & \multicolumn{2}{|c|}{67} \\
\hline range & & & $45-$ & & $37-$ & 120 \\
\hline
\end{tabular}

*EGFR mutations include exon 19 deletion and exon 21 L858R mutation. Pem: pemetrexed; Pem + Bev: pemetrexed plus bevacizumab 
Table 2. Treatment responses

\begin{tabular}{lrrrrrrl}
\hline & $\mathrm{n}$ & $\mathrm{PR}$ & $\mathrm{SD}$ & $\mathrm{PD}$ & $\mathrm{NE}$ & $\mathrm{RR}(\%)$ & $\mathrm{DCR}$ \\
\hline Pem & 20 & 3 & 11 & 4 & 2 & $15^{*}$ & 70 \\
Pem + Bev & 20 & 11 & 7 & 1 & 1 & $55^{*}$ & 95 \\
\hline
\end{tabular}

${ }^{*} \mathrm{p}=0.0146$; Pem: pemetrexed; Pem + Bev: pemetrexed plus bevacizumab; PR: partial response; SD: stable disease; PD: progressive disease; NE: not evaluable; RR: response rate; DCR: disease control rate 
Table 3. Toxicities

\begin{tabular}{|c|c|c|c|c|c|c|}
\hline \multirow[t]{2}{*}{ Adverse event } & \multicolumn{2}{|c|}{ Pem } & \multicolumn{2}{|c|}{$\mathrm{Pem}+\mathrm{Bev}$} & \multicolumn{2}{|c|}{$P$-value } \\
\hline & All (\%) & G3/4 (\%) & All (\%) & G3/4 (\%) & All (\%) & $\mathrm{G} 3 / 4(\%)$ \\
\hline \multicolumn{7}{|l|}{ Hematologic } \\
\hline Leukopenia & $12(60)$ & $2(10)$ & $15(75)$ & $6(30)$ & 0.500 & 0.235 \\
\hline Neutropenia & $13(65)$ & $4(20)$ & $16(80)$ & $11(55)$ & 0.479 & 0.050 \\
\hline Anemia & $18(90)$ & $4(20)$ & $19(95)$ & $1(5)$ & 1.000 & 0.342 \\
\hline Thrombocytopenia & $17(85)$ & $1(5)$ & $16(80)$ & $1(5)$ & 1.000 & 1.000 \\
\hline \multicolumn{7}{|l|}{ Non-hematologic } \\
\hline Nausea & $6(30)$ & $1(5)$ & $7(35)$ & $0(0)$ & 1.000 & 1.000 \\
\hline Vomiting & $2(10)$ & $0(0)$ & $3(15)$ & $0(0)$ & 1.000 & 1.000 \\
\hline Anorexia & $12(60)$ & $1(5)$ & $15(75)$ & $1(5)$ & 0.500 & 1.000 \\
\hline Malaise & $14(70)$ & $0(0)$ & $17(85)$ & $0(0)$ & 0.451 & 1.000 \\
\hline Diarrhea & $1(5)$ & $0(0)$ & $5(25)$ & $0(0)$ & 0.182 & 1.000 \\
\hline Constipation & $4(20)$ & $0(0)$ & $6(30)$ & $0(0)$ & 0.715 & 1.000 \\
\hline Oral mucositis & $0(0)$ & $0(0)$ & $5(25)$ & $1(5)$ & $0.047^{*}$ & 1.000 \\
\hline Alopecia & $0(0)$ & $0(0)$ & $1(5)$ & $0(0)$ & 1.000 & 1.000 \\
\hline Exanthema & $3(15)$ & $1(5)$ & $0(0)$ & $0(0)$ & 0.231 & 1.000 \\
\hline Pneumonitis & $2(10)$ & $0(0)$ & $1(5)$ & $0(0)$ & 1.000 & 1.000 \\
\hline Down of PS & $1(5)$ & $1(5)$ & $1(5)$ & $1(5)$ & 1.000 & 1.000 \\
\hline Elevated ALT & $13(65)$ & $1(5)$ & $16(80)$ & $0(0)$ & 0.479 & 1.000 \\
\hline Elevated AST & $17(85)$ & $2(10)$ & $18(90)$ & $0(0)$ & 1.000 & 0.487 \\
\hline Elevated creatinine & $7(35)$ & $1(5)$ & $8(40)$ & $0(0)$ & 1.000 & 1.000 \\
\hline Hyponatremia & $10(50)$ & $4(20)$ & $18(90)$ & $2(10)$ & $0.014 *$ & 0.661 \\
\hline Hyperkalemia & $8(40)$ & $0(0)$ & $10(50)$ & $1(5)$ & 0.751 & 1.000 \\
\hline Hemosputum & $1(5)$ & $0(0)$ & $1(5)$ & $0(0)$ & 1.000 & 1.000 \\
\hline Proteinuria** & $6(33)$ & $1(6)$ & $11(55)$ & $2(10)$ & 0.201 & 1.000 \\
\hline Hypertension & $0(0)$ & $0(0)$ & $0(0)$ & $0(0)$ & 1.000 & 1.000 \\
\hline Thrombosis & $0(0)$ & $0(0)$ & $0(0)$ & $0(0)$ & 1.000 & 1.000 \\
\hline Hemorrhage & $0(0)$ & $0(0)$ & $1(5)$ & $0(0)$ & 1.000 & 1.000 \\
\hline
\end{tabular}

*Significant difference

**Proteinuria: "All” include $(+),(2+)$ and $(>2+)$, "G3/4" include $(2+)$ and $(>2+)$

Pem: pemetrexed; Pem + Bev: pemetrexed plus bevacizumab; G3/4: grade 3/4; PS: performance status; ALT: alanine aminotransferase; AST: aspartate transaminase 


\section{Supplement Fig. 1}

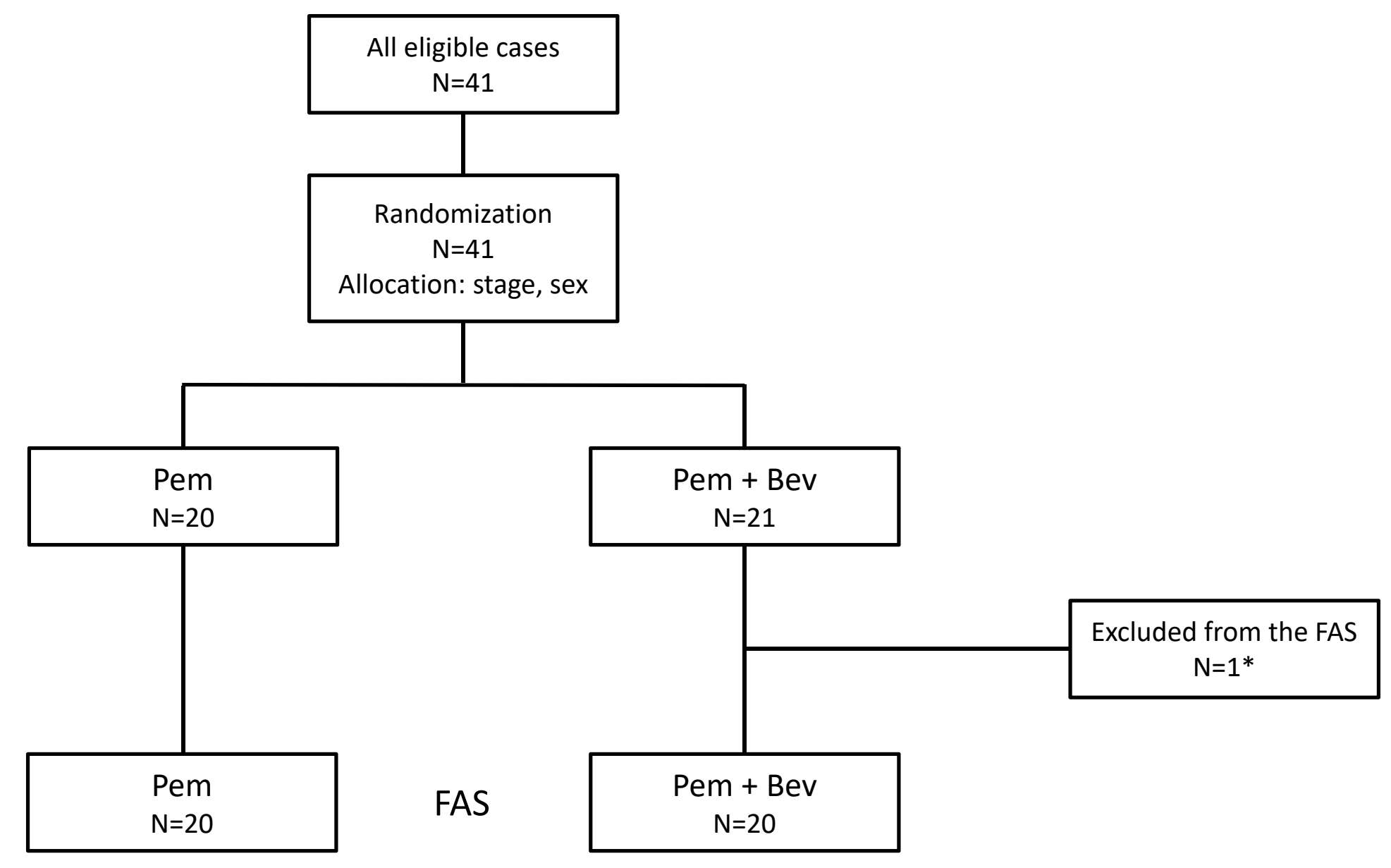




\section{Supplement Fig. 2A}

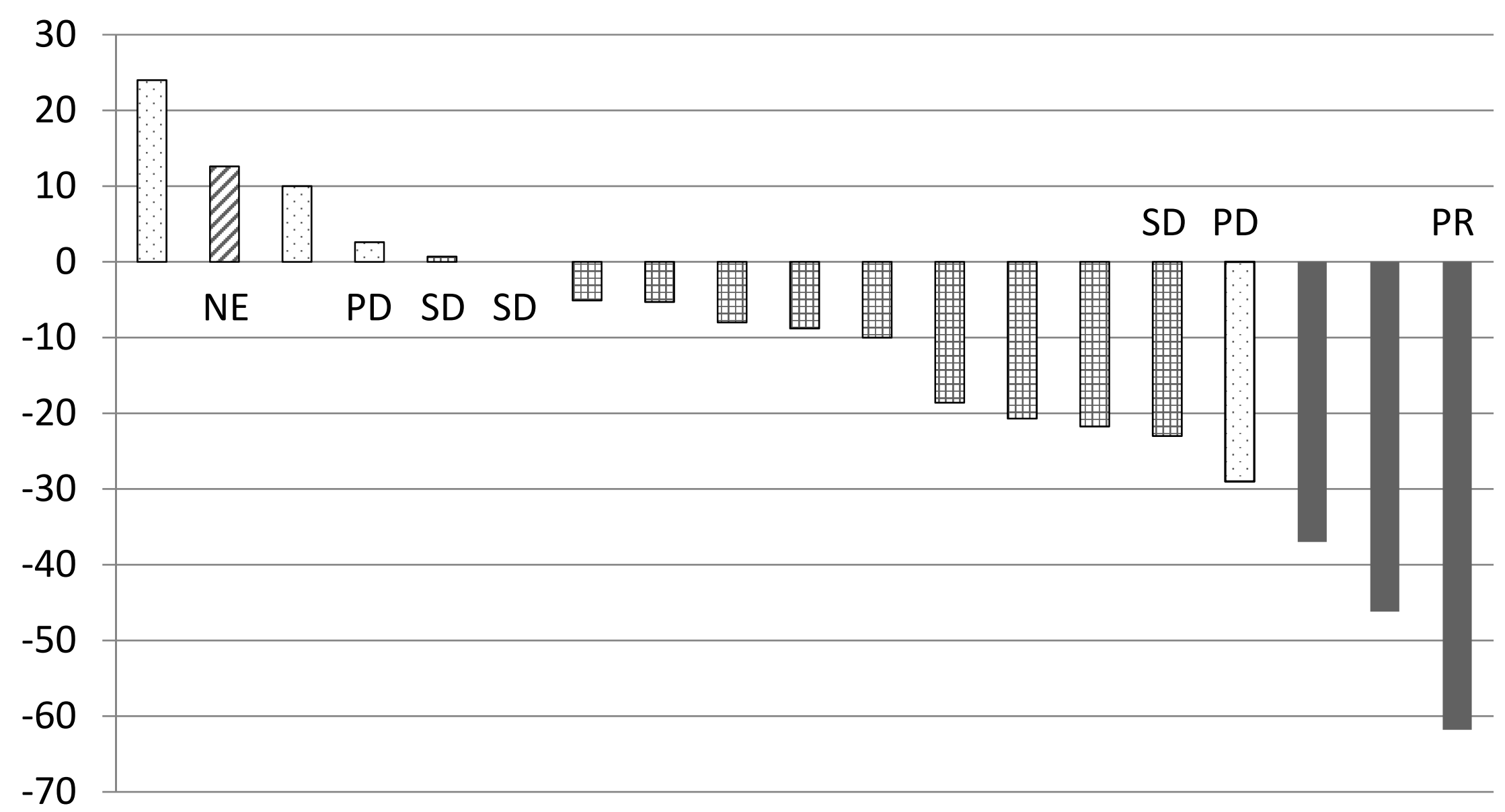




\section{Supplement Fig. 2B}

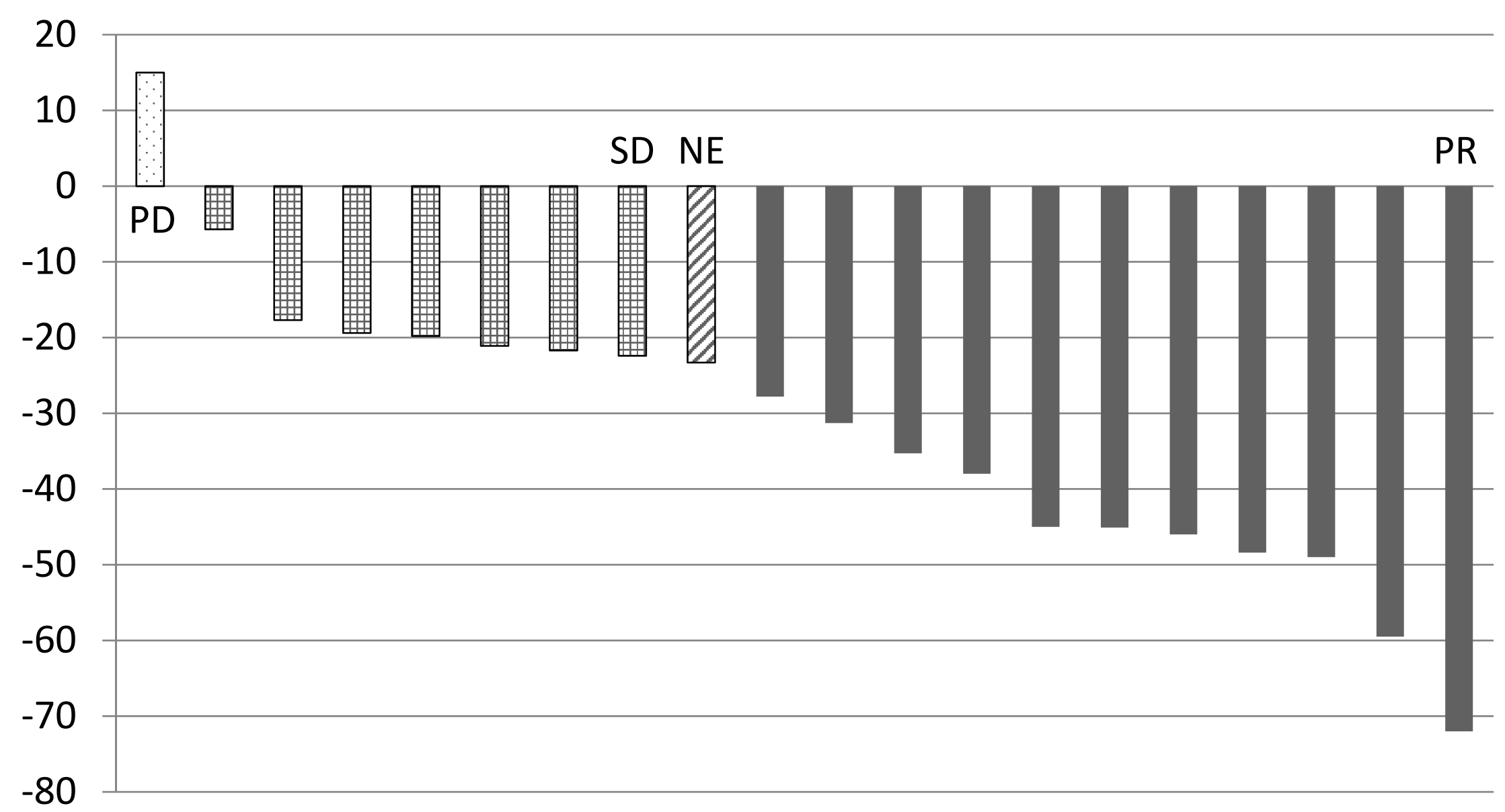




\section{Supplement Fig. 3}

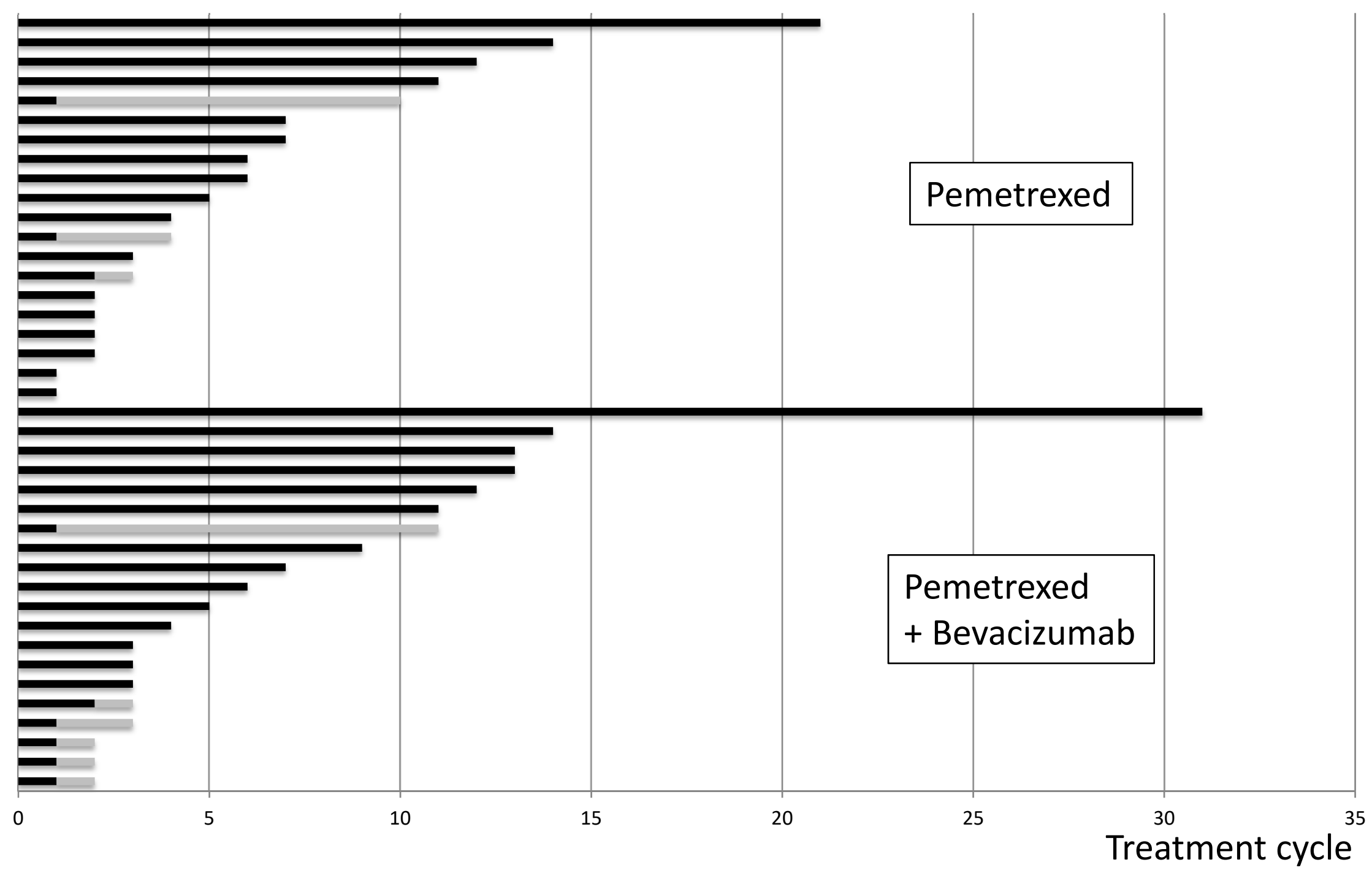




\section{Supplement Table 1}

Table 4. Phase II studies of pemetrexed with or without bevacizumab for elderly patients with NSqNSCLC

\begin{tabular}{lccccccc}
\hline Investigator & age & regimen & $\begin{array}{c}\text { No. of } \\
\text { pts }\end{array}$ & $\begin{array}{c}\text { PFS } \\
\text { (months) }\end{array}$ & $\begin{array}{c}\text { OS } \\
\text { (months) }\end{array}$ & $\begin{array}{c}\text { RR } \\
(\%)\end{array}$ & $\begin{array}{c}\text { G3/4 neutropenia } \\
(\%)\end{array}$ \\
\hline Kim $^{+}$ & $\geq 75$ & Pem & 28 & 3.3 & 17.5 & 25 & 29 \\
Hattori $^{2}$ & $\geq 75$ & Pem & 46 & 4.9 & 18.2 & 13 & 39 \\
Kozuki $^{2}$ & $\geq 70$ & Pem+Bev & 12 & 5.4 & 13.6 & 25 & 25 \\
Schuette $^{3}$ & $\geq 65$ & Pem+Bev & 119 & 4.8 & 11.6 & 31 & 21 \\
Present study & $\geq 75$ & Pem & 20 & 5.4 & 16.0 & 15 & 20 \\
& $\geq 75$ & Pem+Bev & 20 & 5.5 & 16.4 & 55 & 55 \\
\hline
\end{tabular}

NSqNSCLC: non-squamous non-small cell lung cancer; Pem: pemetrexed; Pem + Bev: pemetrexed plus bevacizumab; PFS: progression-free survival; OS: overall survival; RR: response rate; G3/4: grade $3 / 4$ 